\title{
EXPONENTIAL DECAY TO EQUILIBRIUM FOR A FIBER LAY-DOWN PROCESS ON A MOVING CONVEYOR BELT*
}

\author{
EMERIC BOUIN ${ }^{\dagger}$, FRANCA HOFFMANN ${ }^{\ddagger}$, AND CLÉMENT MOUHOT M $^{\S}$
}

\begin{abstract}
We show the existence and uniqueness of a stationary state for a kinetic FokkerPlanck equation modeling the fiber lay-down process in the production of nonwoven textiles. Following a micro-macro decomposition, we use hypocoercivity techniques to show exponential convergence to equilibrium with an explicit rate assuming the conveyor belt moves slowly enough. This work is an extension of [Dolbeault et al., Appl. Math. Res. Express. AMRX, 2013 (2013), pp. 165-175], where the authors consider the case of a stationary conveyor belt. Adding the movement of the belt, the global Gibbs state is not known explicitly. We thus derive a more general hypocoercivity estimate from which existence, uniqueness, and exponential convergence can be derived. To treat the same class of potentials as in [Dolbeault et al., Appl. Math. Res. Express. AMRX, (2013), pp. 165-175] we make use of an additional weight function following the Lyapunov functional approach in [M. Kolb, M. Savov, and A. Wübker, SIAM J. Math. Anal., 45 (2013), pp. 1-13].
\end{abstract}

Key words. hypocoercivity, rate of convergence, fiber lay-down, existence and uniqueness of a stationary state, perturbation, moving belt

AMS subject classifications. 35B20, 35B40, 35B45, 35Q84

DOI. $10.1137 / 16 \mathrm{M} 1077490$

1. Introduction. The mathematical analysis of the fiber lay-down process in the production of nonwoven textiles has seen a lot of interest in recent years [14, $15,6,10,11,4,12]$. Nonwoven materials are produced in melt-spinning operations: hundreds of individual endless fibers are obtained by continuous extrusion through nozzles of a melted polymer. The nozzles are densely and equidistantly placed in a row at a spinning beam. The visco-elastic, slender, and in-extensible fibers lay down on a moving conveyor belt to form a web, where they solidify due to cooling air streams. Before touching the conveyor belt, the fibers become entangled and form loops due to highly turbulent air flow. In [14] a general mathematical model for the fiber dynamics is presented which enables the full simulation of the process. Due to the huge amount of physical details, these simulations of the fiber spinning and lay-down usually require an extremely large computational effort and high memory storage; see [15]. Thus, a simplified two-dimensional stochastic model for the fiber lay-down process, together with its kinetic limit, is introduced in [6]. Generalizations of the two-dimensional stochastic model [6] to three dimensions have been developed by Klar, Maringer, and Wegener in [10] and to any dimension $d \geq 2$ by Grothaus et al. in [7].

${ }^{*}$ Received by the editors June 22, 2016; accepted for publication (in revised form) June 19, 2017; published electronically August 29, 2017.

http://www.siam.org/journals/sima/49-4/M107749.html

Funding: The first and third authors acknowledge the support of the ERC grant MATKIT (ERC-2011-StG). The second author acknowledges support from the Engineering and Physical Sciences Research Council (UK) grant EP/H023348/1 for the University of Cambridge Centre for Doctoral Training, the Cambridge Centre for Analysis.

${ }^{\dagger}$ UMR CNRS 7534, CEREMADE-Université Paris-Dauphine, 75775 Paris Cedex 16, France (bouin@ceremade.dauphine.fr).

${ }^{\ddagger}$ CCA, Centre for Mathematical Sciences, University of Cambridge, Cambridge CB3 0WA, UK (fkoh2@cam.ac.uk).

$\S$ DPMMS, Centre for Mathematical Sciences, University of Cambridge, Cambridge CB3 0WA, UK (c.mouhot@dpmms.cam.ac.uk). 
We now describe the model we are interested in, which comes from [6]. We track the position $x(t) \in \mathbb{R}^{2}$ and the angle $\alpha(t) \in \mathbb{S}^{1}$ of the fiber at the lay-down point where it touches the conveyor belt. Interactions of neighboring fibers are neglected. If $x_{0}(t)$ is the lay-down point in the coordinate system following the conveyor belt, then the tangent vector of the fiber is denoted by $\tau(\alpha(t))$ with $\tau(\alpha)=(\cos \alpha$, $\sin \alpha)$. Since the extrusion of fibers happens at a constant speed, and the fibers are in-extensible, the lay-down process can be assumed to happen at constant normalized speed $\left\|x_{0}^{\prime}(t)\right\|=1$. If the conveyor belt moves with constant speed $\kappa$ in direction $e_{1}=(1,0)$, then

$$
\frac{\mathrm{d} x}{\mathrm{~d} t}=\tau(\alpha)+\kappa e_{1} .
$$

Note that the speed of the conveyor belt cannot exceed the lay-down speed: $0 \leq \kappa \leq 1$. The fiber dynamics in the deposition region close to the conveyor belt are dominated by the turbulent air flow. Applying this concept, the dynamics of the angle $\alpha(t)$ can be described by a deterministic force moving the lay-down point toward the equilibrium $x=0$ and by a Brownian motion modeling the effect of the turbulent air flow. We obtain the following stochastic differential equation for the random variable $X_{t}=\left(x_{t}, \alpha_{t}\right)$ on $\mathbb{R}^{2} \times \mathbb{S}^{1}:$

$$
\left\{\begin{array}{l}
\mathrm{d} x_{t}=\left(\tau\left(\alpha_{t}\right)+\kappa e_{1}\right) \mathrm{d} t, \\
\mathrm{~d} \alpha_{t}=\left[-\tau^{\perp}\left(\alpha_{t}\right) \cdot \nabla_{x} V\left(x_{t}\right)\right] \mathrm{d} t+A \mathrm{~d} W_{t},
\end{array}\right.
$$

where $W_{t}$ denotes a one-dimensional Wiener process, $A>0$ measures its strength relative to the deterministic forcing, $\tau^{\perp}=(-\sin \alpha, \cos \alpha)$, and $V: \mathbb{R}^{2} \longrightarrow \mathbb{R}$ is an external potential carrying information on the coiling properties of the fiber. More precisely, since a curved fiber tends back to its starting point, the change of the angle $\alpha$ is assumed to be proportional to $\tau^{\perp}(\alpha) \cdot \nabla_{x} V(x)$. It has been shown in [12] that under suitable assumptions on the external potential $V$, the fiber lay down process (1) has a unique invariant distribution and is even geometrically ergodic (see Remark 1.2). The stochastic approach yields exponential convergence in total variation norm, but without explicit rate. We will show here that a stronger result can be obtained with a functional analysis approach. Our argument uses crucially the construction of an additional weight functional for the fiber lay-down process in the case of unbounded potential gradients inspired by [12, Proposition 3.7].

The probability density function $f(t, x, \alpha)$ corresponding to the stochastic process (1) is governed by the Fokker-Planck equation

$$
\partial_{t} f+\left(\tau+\kappa e_{1}\right) \cdot \nabla_{x} f-\partial_{\alpha}\left(\tau^{\perp} \cdot \nabla_{x} V f\right)=D \partial_{\alpha \alpha} f
$$

with diffusivity $D=A^{2} / 2$. We state below assumptions on the external potential $V$ that will be used regularly throughout the paper:

(H1) Regularity and symmetry: $V \in C^{2}\left(\mathbb{R}^{2}\right)$ and $V$ is spherically symmetric outside some ball $B\left(0, R_{V}\right)$.

(H2) Normalization: $\int_{\mathbb{R}^{2}} e^{-V(x)} \mathrm{d} x=1$.

(H3) Spectral gap condition (Poincaré inequality): there exists a positive constant $\Lambda$ such that for any $u \in H^{1}\left(e^{-V} \mathrm{~d} x\right)$ with $\int_{\mathbb{R}^{2}} u e^{-V} \mathrm{~d} x=0$,

$$
\int_{\mathbb{R}^{2}}\left|\nabla_{x} u\right|^{2} e^{-V} \mathrm{~d} x \geq \Lambda \int_{\mathbb{R}^{2}} u^{2} e^{-V} \mathrm{~d} x .
$$

Copyright $@$ by SIAM. Unauthorized reproduction of this article is prohibited. 
(H4) Pointwise regularity condition on the potential: there exists $c_{1}>0$ such that for any $x \in \mathbb{R}^{2}$, the Hessian $\nabla_{x}^{2} V$ of $V(x)$ satisfies

$$
\left|\nabla_{x}^{2} V(x)\right| \leq c_{1}\left(1+\left|\nabla_{x} V(x)\right|\right) .
$$

(H5) Behavior at infinity:

$$
\lim _{|x| \rightarrow \infty} \frac{\left|\nabla_{x} V(x)\right|}{V(x)}=0, \quad \lim _{|x| \rightarrow \infty} \frac{\left|\nabla_{x}^{2} V(x)\right|}{\left|\nabla_{x} V(x)\right|}=0 .
$$

Remark 1.1. Assumptions (H2)-(H4) are as stated in [4]. Assumption (H1) assumes regularity of the potential that is stronger and included in that discussed in [4] since (H1) implies $V \in W_{\text {loc }}^{2, \infty}\left(\mathbb{R}^{2}\right)$. Assumption (H5) is only necessary if the potential gradient $\left|\nabla_{x} V\right|$ is unbounded. Both bounded and unbounded potential gradients may appear depending on the physical context, and we will treat these two cases separately where necessary. A typical example for an external potential satisfying assumptions (H1)-(H5) is given by

$$
V(x)=K\left(1+|x|^{2}\right)^{s / 2}
$$

for some constants $K>0$ and $s \geq 1$ [5, 12]. The potential (3) satisfies (H3) since

$$
\liminf _{|x| \rightarrow \infty}\left(\left|\nabla_{x} V\right|^{2}-2 \Delta_{x} V\right)>0
$$

see, for instance, [17, Appendix A.19, p. 135]. The other assumptions are trivially satisfied as can be checked by direct inspection. In this family of potentials, the gradient $\nabla_{x} V$ is bounded for $s=1$ and unbounded for $s>1$.

REMARK 1.2. The proof of ergodicity in [12] assumes that the potential satisfies

$$
\lim _{|x| \rightarrow \infty} \frac{\left|\nabla_{x} V(x)\right|}{V(x)}=0, \quad \lim _{|x| \rightarrow \infty} \frac{\left|\nabla_{x}^{2} V(x)\right|}{\left|\nabla_{x} V(x)\right|}=0, \quad \lim _{|x| \rightarrow \infty}\left|\nabla_{x} V(x)\right|=\infty .
$$

Under these assumptions, there exists an invariant distribution $\nu$ to the fiber lay-down process (1), and some constants $C\left(x_{0}\right)>0, \lambda>0$ such that

$$
\left\|\mathcal{P}_{x_{0}, \alpha_{0}}\left(X_{t} \in \cdot\right)-\nu\right\|_{T V} \leq C\left(x_{0}\right) e^{-\lambda t}
$$

where $\|\cdot\|_{T V}$ denotes the total variation norm. The stochastic Lyapunov technique applied in [12] however does not give any information on how the constant $C\left(x_{0}\right)$ depends on the initial position $x_{0}$ or how the rate of convergence $\lambda$ depends on the conveyor belt speed $\kappa$, the potential $V$, and the noise strength $A$. This can be achieved using hypocoercivity techniques, proving convergence in a weighted $L^{2}$-norm, which is slightly stronger than the convergence in total variation norm shown in [12]. Conceptually, the conditions (4) ensure that the potential $V$ is driving the process back inside a compact set where the noise can be controlled. Our framework (H1)-(H5) is more general than conditions (4) in some aspects (including bounded potential gradient) and more restrictive in others (assuming a Poincaré inequality). The proof in [12] relies on the strong Feller property which can be translated in some cases into a spectral gap; it also uses hypoellipticity to deduce the existence of a transition density and concludes via an explicit Lyapunov function argument. With our framework (H1)(H5), and adapting the Lyapunov function argument presented in [12] to control the effect of $\kappa \partial_{x_{1}}$, we derive an explicit rate of convergence in terms of $\kappa, D$, and $V$. 
To set up a functional framework, rewrite (2) as

$$
\partial_{t} f=\mathrm{L}_{\kappa} f=(\mathrm{Q}-\mathrm{T}) f+\mathrm{P}_{\kappa} f,
$$

where the collision operator $\mathrm{Q}:=D \partial_{\alpha \alpha}$ acts as a multiplicator in the space variable $x, \mathrm{P}_{\kappa}$ is the perturbation introduced by the moving belt with respect to [4],

$$
\mathrm{P}_{\kappa} f:=-\kappa e_{1} \cdot \nabla_{x} f
$$

and the transport operator $\mathrm{T}$ is given by

$$
\mathrm{T} f:=\tau \cdot \nabla_{x} f-\partial_{\alpha}\left(\tau^{\perp} \cdot \nabla_{x} V f\right) .
$$

We consider solutions to (5) in the space $L^{2}\left(\mathrm{~d} \mu_{\kappa}\right):=L^{2}\left(\mathbb{R}^{2} \times \mathbb{S}^{1}, \mathrm{~d} \mu_{\kappa}\right)$ with measure

$$
\mathrm{d} \mu_{\kappa}(x, \alpha)=\left(e^{V(x)}+\zeta \kappa g(x, \alpha)\right) \frac{\mathrm{d} x \mathrm{~d} \alpha}{2 \pi} .
$$

We denote by $\langle\cdot, \cdot\rangle_{\kappa}$ the corresponding scalar product and by $\|\cdot\|_{\kappa}$ the associated norm. Here, $\zeta>0$ is a free parameter to be chosen later. The construction of the weight $g$ depends on the boundedness of $\nabla_{x} V$. When it is bounded, no additional weight is needed to control the perturbation, and so we simply set $g \equiv 0$ in that case. When the gradient is unbounded, the weight is constructed thanks to the following proposition.

Proposition 1.3. Assume that $V$ satisfies (H1) and (H5) and that

$$
\lim _{|x| \rightarrow \infty}\left|\nabla_{x} V\right|=+\infty
$$

If $\kappa<1 / 3$ holds true, then there exists a function $g(x, \alpha)$, a constant $c=c(\kappa, D)>0$, and a finite radius $R=R(k, D, V)>0$ such that

$$
\forall|x|>R, \forall \alpha \in \mathbb{S}^{1}, \quad \mathcal{L}_{\kappa}(g)(x, \alpha) \leq-c\left|\nabla_{x} V(x)\right| g(x, \alpha),
$$

where $\mathcal{L}_{\kappa}$ is defined by

$$
\mathcal{L}_{\kappa}(h):=D \partial_{\alpha \alpha} h+\left(\tau+\kappa e_{1}\right) \cdot \nabla_{x} h-\left(\tau^{\perp} \cdot \nabla_{x} V\right) \partial_{\alpha} h-\left(\tau \cdot \nabla_{x} V\right) h .
$$

The weight $g$ is of the form

$$
g(x, \alpha):=\exp \left(\beta V(x)+\left|\nabla_{x} V(x)\right| \Gamma\left(\tau(\alpha) \cdot \frac{\nabla_{x} V(x)}{\left|\nabla_{x} V(x)\right|}\right)\right),
$$

where the parameter $\beta>1$ and the function $\Gamma \in C^{1}([-1,1]), \Gamma>0$ are determined along the proof and only depend on $\kappa$.

We show in section 3 the existence of such a weight function $g$ under appropriate conditions following ideas from [12].

We denote $\mathcal{C}:=C_{c}^{\infty}\left(\mathbb{R}^{2} \times \mathbb{S}^{1}\right)$ and define the orthogonal projection $\Pi$ on the set of local equilibria $\operatorname{Ker} Q$

$$
\Pi f:=\int_{\mathbb{S}^{1}} f \frac{\mathrm{d} \alpha}{2 \pi}
$$

and the mass $M_{f}$ of a given distribution $f \in L^{2}\left(\mathrm{~d} \mu_{\kappa}\right)$

$$
M_{f}:=\int_{\mathbb{R}^{2} \times \mathbb{S}^{1}} f \frac{\mathrm{d} x \mathrm{~d} \alpha}{2 \pi} .
$$

Copyright $\odot$ by SIAM. Unauthorized reproduction of this article is prohibited. 
Integrating (2) over $\mathbb{R}^{2} \times \mathbb{S}^{1}$ shows that the mass of solutions of (2) is conserved over time, and standard maximum principle arguments show that it remains nonnegative for nonnegative initial data. The collision operator $Q$ is symmetric and satisfies

$$
\forall f \in \mathcal{C}, \quad\langle Q f, f\rangle_{0}=-D\left\|\partial_{\alpha} f\right\|_{0}^{2} \leq 0,
$$

i.e., $\mathbf{Q}$ is dissipative in $L^{2}\left(\mathrm{~d} \mu_{0}\right)$. Further, we have $\mathrm{T} \Pi f=e^{-V} \tau \cdot \nabla_{x} u_{f}$ for $f \in \mathcal{C}$ with $u_{f}:=e^{V} \Pi f$, which implies $\Pi \mathrm{T} \Pi=0$ on $\mathcal{C}$. Since the transport operator $\mathrm{T}$ is skew-symmetric with respect to $\langle\cdot, \cdot\rangle_{0}$,

$$
\left\langle\mathrm{L}_{\kappa} f, f\right\rangle_{0}=\langle\mathrm{Q} f, f\rangle_{0}+\left\langle\mathrm{P}_{\kappa} f, f\right\rangle_{0}
$$

for any $f$ in $\mathcal{C}$. In the case $\kappa=0$, if the entropy dissipation $-\langle Q f, f\rangle_{0}$ was coercive with respect to the norm $\|\cdot\|_{0}$, exponential decay to zero would follow as $t \rightarrow \infty$. However, such a coercivity property cannot hold since $Q$ vanishes on the set of local equilibria. Instead, Dolbeault, Mouhot, and Schmeiser [5] applied a strategy called hypocoercivity (as theorized in [17]) and developed by several groups in the 2000s; see, for instance, $[9,8,13,2,3]$. The full hypocoercivity analysis of the long time behavior of solutions to this kinetic model in the case of a stationary conveyor belt, $\kappa=0$, is completed in [4]. For technical applications in the production process of nonwovens, one is interested in a model including the movement of the conveyor belt, and our aim is to extend the results in [4] to small $\kappa>0$.

We follow the approach of hypocoercivity for linear kinetic equations conserving mass developed in [5], with several new difficulties. Considering the case $\kappa=0, \mathrm{Q}$ and $\mathrm{T}$ are closed operators on $L^{2}\left(\mathrm{~d} \mu_{0}\right)$ such that $\mathrm{Q}-\mathrm{T}$ generates the $\mathcal{C}_{0}$-semigroup $e^{(\mathbf{Q}-\mathrm{T}) t}$ on $L^{2}\left(\mathrm{~d} \mu_{0}\right)$. When $\kappa>0$, we use the additional weight function $g>0$ to control the perturbative term $\mathrm{P}_{\kappa}$ in the case of unbounded potential gradients and show the existence of a $\mathcal{C}_{0}$-semigroup for $\mathrm{L}_{\kappa}=\mathrm{Q}-\mathrm{T}+\mathrm{P}_{\kappa}$ (see section 4.1). Unless otherwise specified, all computations are performed on the operator core $\mathcal{C}$ and can be extended to $L^{2}\left(\mathrm{~d} \mu_{\kappa}\right)$ by density arguments.

When $\kappa=0$, the hypocoercivity result in $[5,4]$ is based on microscopic coercivity, which assumes that the restriction of $\mathrm{Q}$ to $(\mathrm{Ker} \mathrm{Q})^{\perp}$ is coercive, and macroscopic coercivity, which is a spectral gap-like inequality for the operator obtained when taking a parabolic drift-diffusion limit - in other words, the restriction of $T$ to Ker Q is coercive. The two properties are satisfied in the case of a stationary conveyor belt:

- The operator $Q$ is symmetric and the Poincaré inequality on $\mathbb{S}^{1}$,

$$
\frac{1}{2 \pi} \int_{\mathbb{S}^{1}}\left|\partial_{\alpha} f\right|^{2} \mathrm{~d} \alpha \geq \frac{1}{2 \pi} \int_{\mathbb{S}^{1}}\left(f-\frac{1}{2 \pi} \int_{\mathbb{S}^{1}} f \mathrm{~d} \alpha\right)^{2} \mathrm{~d} \alpha
$$

implies that $-\langle\mathrm{Q} f, f\rangle_{0} \geq D\|(1-\Pi) f\|_{0}^{2}$.

- The operator T is skew-symmetric and for any $h \in L^{2}\left(\mathrm{~d} \mu_{0}\right)$ such that $u_{h}=$ $e^{V} \Pi h \in H^{1}\left(e^{-V} \mathrm{~d} x\right)$ and $\int_{\mathbb{R}^{2} \times \mathbb{S}^{1}} h \mathrm{~d} \mu_{0}=0$, (H3) implies

$$
\|\mathrm{T} \Pi h\|_{0}^{2}=\frac{1}{4 \pi} \int_{\mathbb{R}^{2} \times \mathbb{S}^{1}} e^{-V}\left|\nabla_{x} u_{h}\right|^{2} \mathrm{~d} x \mathrm{~d} \alpha \geq \frac{\Lambda}{4 \pi} \int_{\mathbb{R}^{2} \times \mathbb{S}^{1}} e^{-V} u_{h}^{2} \mathrm{~d} x \mathrm{~d} \alpha=\frac{\Lambda}{2}\|\Pi h\|_{0}^{2} .
$$

In the case $\kappa=0$, the unique global normalized equilibrium distribution $F_{0}=e^{-V}$ lies in the intersection of the null spaces of $\mathrm{T}$ and $\mathrm{Q}$. When $\kappa>0, F_{0}$ is not in the kernel of $\mathrm{P}_{\kappa}$ and we are not able to find the global Gibbs state of (5) explicitly. However, the hypocoercivity theory is based on a priori estimates [5] that are, as we shall prove, to some extent stable under perturbation. Our main result reads as follows. 
TheOrem 1.4. Let $f_{\text {in }} \in L^{2}\left(\mathrm{~d} \mu_{\kappa}\right)$ and let (H1-2-3-4-5) hold. For $0<\kappa<1$ small enough (with a quantitative estimate) and $\zeta>0$ large enough (with a quantitative estimate), there exists a unique nonnegative stationary state $F_{\kappa} \in L^{2}\left(\mathrm{~d} \mu_{\kappa}\right)$ with unit mass $M_{F_{\kappa}}=1$. In addition, for any solution $f$ of $(2)$ in $L^{2}\left(\mathrm{~d} \mu_{\kappa}\right)$ with mass $M_{f}$ and subject to the initial condition $f(t=0)=f_{\text {in }}$, we have

$$
\left\|f(t, \cdot)-M_{f} F_{\kappa}\right\|_{\kappa} \leq C\left\|f_{\text {in }}-M_{f} F_{\kappa}\right\|_{\kappa} e^{-\lambda_{\kappa} t},
$$

where the rate of convergence $\lambda_{\kappa}>0$ depends only on $\kappa, D$, and $V$, and the constant $C>0$ depends only on $D$ and $V$.

In the case of a stationary conveyor belt $\kappa=0$ considered in [4], the stationary state is characterized by the eigenpair $\left(\Lambda_{0}, F_{0}\right)$ with $\Lambda_{0}=0, F_{0}=e^{-V}$, and so $\operatorname{Ker} \mathrm{L}_{0}=\left\langle F_{0}\right\rangle$. This means that there is an isolated eigenvalue $\Lambda_{0}=0$ and a spectral gap of size at least $\left[-\lambda_{0}, 0\right]$ with the rest of the spectrum $\Sigma\left(L_{0}\right)$ to the left of $-\lambda_{0}$ in the complex plane. Adding the movement of the conveyor belt, Theorem 1.4 shows that $\operatorname{Ker} \mathrm{L}_{\kappa}=\left\langle F_{\kappa}\right\rangle$ and the exponential decay to equilibrium with rate $\lambda_{\kappa}$ corresponds to a spectral gap of size at least $\left[-\lambda_{\kappa}, 0\right]$. Further, it allows one to recover an explicit expression for the rate of convergence $\lambda_{0}$ for $\kappa=0$ (see Step 5 in section 2.1). In general, we are not able to compute the stationary state $F_{\kappa}$ for $\kappa>0$ explicitly, but $F_{\kappa}$ converges to $F_{0}=e^{-V}$ weakly as $\kappa \rightarrow 0$. (See the discussion in section 5.) Let us finally emphasize that a specific contribution of our paper is to introduce two (and not one as in [5, 4]) modifications of the entropy: (1) we first modify the space itself with the coercivity weight $g$, and then (2) we change the norm with an auxiliary operator following the hypocoercivity approach.

The rest of the paper deals with the case $\kappa>0$ and is organized as follows. In section 2 , we prove the main hypocoercivity estimate. This allows us to establish the existence of solutions to (2) using semigroup theory and to deduce the existence and uniqueness of a steady state in section 4 by a contraction argument. In section 3 , we give a detailed definition of the weight function $g$ that is needed for the hypocoercivity estimate in section 2 .

2. Hypocoercivity estimate. Following [5] we introduce the auxiliary operator

$$
\mathrm{A}:=\left(1+(\mathrm{T \Pi})^{*}(\mathrm{~T} \Pi)\right)^{-1}(\mathrm{~T} \Pi)^{*}
$$

and a modified entropy, i.e., a hypocoercivity functional $\mathrm{G}$ on $L^{2}\left(\mathrm{~d} \mu_{\kappa}\right)$ :

$$
\mathrm{G}[f]:=\frac{1}{2}\|f\|_{\kappa}^{2}+\varepsilon_{1}\langle\mathrm{~A} f, f\rangle_{0}, \quad f \in L^{2}\left(\mathrm{~d} \mu_{\kappa}\right)
$$

for some suitably chosen $\varepsilon_{1} \in(0,1)$ to be determined later. It follows from [5] that $\left|\langle\mathrm{A} f, f\rangle_{0}\right| \leq\|f\|_{0}^{2}$. Also, $\|f\|_{0}^{2} \leq\|f\|_{\kappa}^{2}$ by construction of $\mu_{\kappa}$, and hence G[·] is normequivalent to $\|\cdot\|_{\kappa}^{2}$ :

$$
\forall f \in L^{2}\left(\mathrm{~d} \mu_{\kappa}\right), \quad\left(\frac{1-\varepsilon_{1}}{2}\right)\|f\|_{\kappa}^{2} \leq \mathrm{G}[f] \leq\left(\frac{1+\varepsilon_{1}}{2}\right)\|f\|_{\kappa}^{2} .
$$

In this section, we prove the following hypocoercivity estimate.

Proposition 2.1. Assume that hypothesis (H1)-(H5) hold and that $0<\kappa<1$ is small enough (with a quantitative estimate). Let $f_{\mathrm{in}} \in L^{2}\left(\mathrm{~d} \mu_{\kappa}\right)$ and $f=f(t, x, \alpha)$ be a solution of $(2)$ in $L^{2}\left(\mathrm{~d} \mu_{\kappa}\right)$ subject to the initial condition $f(t=0)=f_{\mathrm{in}}$. Then $f$ 
satisfies the following Grönwall type estimate:

$$
\frac{\mathrm{d}}{\mathrm{d} t} \mathrm{G}[f(t, \cdot)] \leq-\gamma_{1} \mathrm{G}[f(t, \cdot)]+\gamma_{2} M_{f}^{2}
$$

where $\gamma_{1}>0, \gamma_{2}>0$ are explicit constants only depending on $\kappa, D$, and $V$.

Note that the estimate (10) is stronger than what is required for the uniqueness of a global Gibbs state and represents an extension of the estimate given in [4]. When applied to the difference of two solutions with the same mass, (10) gives an estimate on the exponential decay rate towards equilibrium.

2.1. Proof of Proposition 2.1. Differentiate in time G $[f]$ to get

$$
\frac{\mathrm{d}}{\mathrm{d} t} \mathrm{G}[f]=\mathrm{D}_{0}[f]+\mathrm{D}_{1}[f]+\mathrm{D}_{2}[f]+\mathrm{D}_{3}[f],
$$

where the entropy dissipation functionals $\mathrm{D}_{0}, \mathrm{D}_{1}, \mathrm{D}_{2}$, and $\mathrm{D}_{3}$ are given by

$$
\begin{aligned}
\mathrm{D}_{0}[f]:= & \langle\mathrm{Q} f, f\rangle_{0}-\varepsilon_{1}\langle\operatorname{AT} \Pi f, \Pi f\rangle_{0}-\varepsilon_{1}\langle\mathrm{AT}(1-\Pi) f, \Pi f\rangle_{0} \\
& +\varepsilon_{1}\langle\mathrm{TA} f,(1-\Pi) f\rangle_{0}+\varepsilon_{1}\langle\mathrm{AQ} f, \Pi f\rangle_{0}, \\
\mathrm{D}_{1}[f]:= & \varepsilon_{1}\left\langle\mathrm{AP}_{\kappa} f, \Pi f\right\rangle_{0}+\varepsilon_{1}\left\langle\mathrm{P}_{\kappa}^{*} \mathrm{~A} f, \Pi f\right\rangle_{0}, \\
\mathrm{D}_{2}[f]:= & \left\langle\mathrm{P}_{\kappa} f, f\right\rangle_{0}, \\
\mathrm{D}_{3}[f]:= & \kappa \zeta \int_{\mathbb{R}^{2} \times \mathbb{S}^{1}} \mathrm{~L}_{\kappa}(f) f g \frac{\mathrm{d} x \mathrm{~d} \alpha}{2 \pi} .
\end{aligned}
$$

Note that the term $\langle\text { LA } f, f\rangle_{0}$ vanishes since it has been shown in [5] that $\mathrm{A}=\Pi \mathrm{A}$ and hence A $f \in \operatorname{Ker} \mathrm{Q}$. Further, $\langle\mathrm{T} f, f\rangle=0$ since $\mathrm{T}$ is skew-symmetric. We estimate the entropy dissipation of the case $\kappa=0$ as in [4]:

Step 1: Estimation of $\mathrm{D}_{0}[f]$.

We will show the boundedness of $D_{0}$, which is in fact the dissipation functional for a stationary conveyor belt. We thus recall without proof in the following lemma some results from [4].

Lemma 2.2 (Dolbeault et al. [4]). The following estimates hold:

$$
\begin{aligned}
\langle\mathrm{Q} f, f\rangle_{0} & \leq-\|(1-\Pi) f\|_{0}^{2}, & \|\mathrm{AT}(1-\Pi) f\|_{0} & \leq C_{V}\|(1-\Pi) f\|_{0}, \\
\|\mathrm{AQ} f\|_{0} & \leq \frac{D}{2}\|(1-\Pi) f\|_{0}, & \|\mathrm{TA} f\|_{0} & \leq\|(1-\Pi) f\|_{0} .
\end{aligned}
$$

In order to control the contribution $\langle\operatorname{AT} \Pi f, \Pi f\rangle_{0}$ in $\mathrm{D}_{0}$, we note that

$$
\mathrm{AT \Pi}=\left(1+(\mathrm{T} \Pi)^{*} \mathrm{~T} \Pi\right)^{-1}(\mathrm{~T} \Pi)^{*} \mathrm{~T} \Pi
$$

shares its spectral decomposition with (ТП)*ТП, and by macroscopic coercivity

$$
\left\langle(\mathrm{T} \Pi)^{*} \mathrm{~T} \Pi f, f\right\rangle_{0}=\|\mathrm{T} \Pi f\|_{0}^{2}=\left\|\mathrm{T} \Pi\left(f-M_{f} e^{-V}\right)\right\|_{0}^{2} \geq \frac{\Lambda}{2}\left\|\Pi\left(f-M_{f} e^{-V}\right)\right\|_{0}^{2} .
$$

Hence,

$$
\langle\operatorname{AT} \Pi f, f\rangle_{0} \geq \frac{\Lambda / 2}{1+\Lambda / 2}\left\|\Pi\left(f-M_{f} e^{-V}\right)\right\|_{0}^{2}
$$

Copyright (c) by SIAM. Unauthorized reproduction of this article is prohibited. 
Now, recalling Lemma 2.2 and using $\left\|\Pi\left(f-M_{f} e^{-V}\right)\right\|_{0}^{2}=\|\Pi f\|_{0}^{2}-M_{f}^{2}$, we estimate

$$
\mathrm{D}_{0}[f] \leq\left(\varepsilon_{1}-D\right)\|(1-\Pi) f\|_{0}^{2}+\varepsilon_{1} \lambda_{2}\|(1-\Pi) f\|_{0}\|\Pi f\|_{0}-\varepsilon_{1} \gamma_{2}\left(\|\Pi f\|_{0}^{2}-M_{f}^{2}\right)
$$

with $\lambda_{2}:=C_{V}+D / 2>0$ and $\gamma_{2}:=\frac{\Lambda / 2}{1+\Lambda / 2}>0$.

Step 2: Estimation of $\mathrm{D}_{1}[f]$.

We now turn to the entropy dissipation functional $D_{1}$, which we will estimate using elliptic regularity. Instead of bounding $\mathrm{AP}_{\kappa}$, we apply an elliptic regularity strategy to its adjoint, as for AT $(1-\Pi)$ in [4]. Let $f \in L^{2}\left(\mathrm{~d} \mu_{0}\right)$ and define $h:=\left(1+(\mathrm{T} \Pi)^{*} \mathrm{~T} \Pi\right)^{-1} f$ so that $u_{h}=e^{V} \Pi h$ satisfies

$$
\Pi f=e^{-V} u_{h}+\Pi \mathbf{T}^{*} \mathrm{~T}\left(e^{-V} u_{h}\right)=e^{-V} u_{h}-\frac{1}{2} \nabla_{x} \cdot\left(e^{-V} \nabla_{x} u_{h}\right) .
$$

We have used here the fact that in the space $L^{2}\left(\mathrm{~d} \mu_{0}\right)$,

$$
\left\{\begin{array}{l}
\mathbf{T}=\tau \cdot \nabla_{x}-\partial_{\alpha}\left[\left(\tau^{\perp} \cdot \nabla_{x} V\right)\right], \\
\mathbf{T}^{*}=-\tau \cdot \nabla_{x}+\left(\tau^{\perp} \cdot \nabla_{x} V\right) \partial_{\alpha}-\left(\tau \cdot \nabla_{x} V\right) .
\end{array}\right.
$$

Then

$$
\mathrm{A}^{*} f=\mathrm{T} \Pi h=e^{-V} \tau \cdot \nabla_{x} u_{h},
$$

and since the adjoint for $\langle\cdot, \cdot\rangle_{0}$ of the perturbation operator $\mathrm{P}_{\kappa}$ is given by

$$
\mathrm{P}_{\kappa}^{*}=-\mathrm{P}_{\kappa}-\mathrm{P}_{\kappa} V
$$

it follows that

$$
\begin{aligned}
\left\|\left(\mathrm{AP}_{\kappa}\right)^{*} f\right\|_{0}^{2} & =\left\|\kappa \tau \cdot \nabla_{x}\left(e_{1} \cdot \nabla_{x} u_{h}\right) e^{-V}\right\|_{0}^{2} \\
& =\frac{\kappa^{2}}{2} \int_{\mathbb{R}^{2} \times \mathbb{S}^{1}} e^{-V}\left|\tau \cdot \nabla_{x}\left(e_{1} \cdot \nabla_{x} u_{h}\right)\right|^{2} \mathrm{~d} \mu_{0} \\
& =\frac{\kappa^{2}}{2} \int_{\mathbb{R}^{2}} e^{-V}\left|\nabla_{x}\left(e_{1} \cdot \nabla_{x} u_{h}\right)\right|^{2} \mathrm{~d} x \\
& \leq \frac{\kappa^{2}}{2}\left\|\nabla_{x}^{2} u_{h}\right\|_{L^{2}\left(e^{-V} \mathrm{~d} x\right)}^{2} \\
& \leq \frac{\kappa^{2}}{2} C_{V}^{2}\|\Pi f\|_{0}^{2},
\end{aligned}
$$

where in the last inequality we have used an elliptic regularity estimate. This estimate turns out to be a particular case of [4, Proposition 5 and sections 2-3], where the positive constant $C_{V}$ is the same as in Lemma 2.2 reproduced from [4]. This concludes the boundedness of $\mathrm{AP}_{\kappa}$,

$$
\left\|\mathrm{AP}_{\kappa} f\right\|_{0} \leq \kappa \frac{C_{V}}{\sqrt{2}}\|\Pi f\|_{0} \leq \kappa \frac{C_{V}}{\sqrt{2}}\|f\|_{0} .
$$

Using a similar approach for the operator $\mathrm{P}_{\kappa}^{*} \mathrm{~A}$, we rewrite its adjoint as

$$
\mathrm{A}^{*} \mathrm{P}_{\kappa} f=\mathrm{T} \Pi \tilde{h}
$$

where we define $\tilde{h}:=\left(1+(\mathrm{T} \Pi)^{*} \mathrm{~T} \Pi\right)^{-1} \mathrm{P}_{\kappa} f$ for a given $f \in L^{2}\left(\mathrm{~d} \mu_{0}\right)$, or equivalently

$$
e^{-V} u_{\tilde{h}}-\frac{1}{2} \nabla_{x} \cdot\left(e^{-V} \nabla_{x} u_{\tilde{h}}\right)=\Pi \mathrm{P}_{\kappa} f=\mathrm{P}_{\kappa} \Pi f .
$$

Copyright (c) by SIAM. Unauthorized reproduction of this article is prohibited. 
Multiplying by $u_{\tilde{h}}$ and integrating over $\mathbb{R}^{2}$, we have

$$
\begin{aligned}
\left\|u_{\tilde{h}}\right\|_{L^{2}\left(e^{-V} \mathrm{~d} x\right)}^{2}+\frac{1}{2}\left\|\nabla_{x} u_{\tilde{h}}\right\|_{L^{2}\left(e^{-V} \mathrm{~d} x\right)}^{2} & =-\kappa \int_{\mathbb{R}^{2}} \mathbf{e}_{1} \cdot \nabla_{x}(\Pi f) u_{\tilde{h}} \mathrm{~d} x \\
& =\kappa \int_{\mathbb{R}^{2}}(\Pi f) \mathbf{e}_{1} \cdot \nabla_{x} u_{\tilde{h}} \mathrm{~d} x \\
& \leq \kappa \int_{\mathbb{R}^{2}}\left|\nabla_{x} u_{\tilde{h}} e^{-V / 2}\right|\left|\Pi f e^{V / 2}\right| \mathrm{d} x \\
& \leq \kappa\left\|\nabla_{x} u_{\tilde{h}}\right\|_{L^{2}\left(e^{-V} \mathrm{~d} x\right)}\|\Pi f\|_{0} \\
& \leq \frac{1}{4}\left\|\nabla_{x} u_{\tilde{h}}\right\|_{L^{2}\left(e^{-V} \mathrm{~d} x\right)}^{2}+\kappa^{2}\|\Pi f\|_{0}^{2} .
\end{aligned}
$$

This inequality is a $H^{1}\left(e^{-V} \mathrm{~d} x\right) \rightarrow H^{-1}\left(e^{-V} \mathrm{~d} x\right)$ elliptic regularity result. Hence,

$$
\left\|\mathrm{A}^{*} \mathrm{P}_{\kappa} f\right\|_{0}^{2}=\|\mathrm{T} \Pi h\|_{0}^{2}=\frac{1}{2}\left\|\nabla_{x} u_{\tilde{h}}\right\|_{L^{2}\left(e^{-V} \mathrm{~d} x\right)}^{2} \leq 2 \kappa^{2}\|\Pi f\|_{0}^{2},
$$

and so we conclude

$$
\left\|\mathrm{P}_{\kappa}^{*} \mathrm{~A} f\right\|_{0} \leq \sqrt{2} \kappa\|(1-\Pi) f\|_{0} \leq \sqrt{2} \kappa\|f\|_{0} .
$$

Combining (11) and (12), the entropy dissipation functional $D_{1}$ is bounded by

$$
\mathrm{D}_{1}[f] \leq \kappa \varepsilon_{1}\left(\frac{C_{V}}{\sqrt{2}}+\sqrt{2}\right)\|f\|_{0}^{2}=2 \kappa \lambda_{1}\|f\|_{0}^{2}
$$

where we defined $\lambda_{1}:=\frac{1}{2}\left(\frac{C_{V}}{\sqrt{2}}+\sqrt{2}\right)$.

Step 3: Estimation of $\mathrm{D}_{2}[f]$.

Using integration by parts, we have

$$
\left\langle\mathrm{P}_{\kappa} f, f\right\rangle_{0}=\frac{\kappa}{2} \int_{\mathbb{R}^{2} \times \mathbb{S}^{1}}\left(e_{1} \cdot \nabla_{x} V\right) f^{2} e^{V} \frac{\mathrm{d} x \mathrm{~d} \alpha}{2 \pi} .
$$

The estimation of this term goes differently depending on the boundedness of $\nabla_{x} V$.

If $\nabla_{x} V$ is bounded, we write

$$
\mathrm{D}_{2}[f] \leq\left|\left\langle\mathrm{P}_{\kappa} f, f\right\rangle_{0}\right| \leq \frac{\kappa}{2}\left\|\nabla_{x} V\right\|_{\infty}\|f\|_{0}^{2}=\frac{\kappa}{2}\left\|\nabla_{x} V\right\|_{\infty}\|f\|_{\kappa}^{2},
$$

where we have used $\|f\|_{\kappa}=\|f\|_{0}$, since $g \equiv 0$.

Assume now that $\left|\nabla_{x} V\right| \rightarrow \infty$ as $|x| \rightarrow \infty$. Thanks to the choice of $g$, we have the estimate

$$
\mathrm{D}_{2}[f] \leq\left|\left\langle\mathrm{P}_{\kappa} f, f\right\rangle_{0}\right| \leq \frac{\kappa}{2} \int_{\mathbb{R}^{2} \times \mathbb{S}^{1}}\left|\nabla_{x} V\right| f^{2} e^{V} \frac{\mathrm{d} x \mathrm{~d} \alpha}{2 \pi} \leq \frac{\kappa}{2} C_{3} \int_{\mathbb{R}^{2} \times \mathbb{S}^{1}} f^{2} g \frac{\mathrm{d} x \mathrm{~d} \alpha}{2 \pi}
$$

with

$$
C_{3}:=\sup _{x \in \mathbb{R}^{2}}\left(\left|\nabla_{x} V\right| e^{V} g^{-1}\right)
$$

which is finite by (H5). 
Step 4: Estimation of $\mathrm{D}_{3}[f]$.

We start by recalling that this estimate is only relevant when $\nabla_{x} V$ is unbounded. Indeed, in the opposite case, $\mathrm{D}_{3}[f]=0$ since $g \equiv 0$ by definition. By the identity

$$
\int_{\mathbb{R}^{2} \times \mathbb{S}^{1}} \mathrm{~L}_{\kappa}(f) f g \mathrm{~d} x \mathrm{~d} \alpha=\frac{1}{2} \int_{\mathbb{R}^{2} \times \mathbb{S}^{1}} \mathcal{L}_{\kappa}(g) f^{2} \mathrm{~d} x \mathrm{~d} \alpha-D \int_{\mathbb{R}^{2} \times \mathbb{S}^{1}}\left|\partial_{\alpha} f\right|^{2} g \mathrm{~d} x \mathrm{~d} \alpha
$$

with $\mathcal{L}_{\kappa}$ as defined in (7), we have

$$
\mathrm{D}_{3}[f] \leq \kappa \zeta\left(\frac{1}{2} \int_{\mathbb{R}^{2} \times \mathbb{S}^{1}} \mathcal{L}_{\kappa}(g) f^{2} \frac{\mathrm{d} x \mathrm{~d} \alpha}{2 \pi}\right) .
$$

Proposition 1.3 allows us to control the $g$-weighted $L^{2}$-norm outside some fixed ball. More precisely, take $R>0$ in (6) large enough s.t. $\left|\nabla_{x} V\right| \geq 1$ for all $|x|>R$, and then

$$
\begin{aligned}
& \int_{\mathbb{R}^{2} \times \mathbb{S}^{1}} \mathcal{L}_{\kappa}(g) f^{2} \frac{\mathrm{d} x \mathrm{~d} \alpha}{2 \pi} \\
& \leq \int_{\mathbb{S}^{1}} \int_{|x|<R} \mathcal{L}_{\kappa}(g) f^{2} \frac{\mathrm{d} x \mathrm{~d} \alpha}{2 \pi}-c \int_{\mathbb{S}^{1}} \int_{|x|>R}\left|\nabla_{x} V\right| f^{2} g \frac{\mathrm{d} x \mathrm{~d} \alpha}{2 \pi} \\
& \leq \int_{\mathbb{S}^{1}} \int_{|x|<R}\left(\left(\mathcal{L}_{\kappa}(g)+c g\right) e^{-V}\right) f^{2} e^{V} \frac{\mathrm{d} x \mathrm{~d} \alpha}{2 \pi}-c \int_{\mathbb{R}^{2} \times \mathbb{S}^{1}} f^{2} g \frac{\mathrm{d} x \mathrm{~d} \alpha}{2 \pi} \\
& \leq C_{4}(R)\|f\|_{0}^{2}-c \int_{\mathbb{R}^{2} \times \mathbb{S}^{1}} f^{2} g \frac{\mathrm{d} x \mathrm{~d} \alpha}{2 \pi}
\end{aligned}
$$

where $C_{4}(R):=\sup _{|x| \leq R}\left(\left|\mathcal{L}_{\kappa}(g)+c g\right| e^{-V}\right)$.

REMARK 2.3. Observe here that one could take advantage of the growth of $\nabla_{x} V$ by playing with the cutoff parameter $R$ and keeping track of $\min _{|x| \geq R}\left|\nabla_{x} V\right|$ in the negative term. It could lead to more optimal constants, but we chose instead to vary the parameter $\zeta$ in front of the coercivity weight $g$ in the measure $\mu_{\kappa}$ for simplicity.

Step 5: Putting the four previous steps together.

Combine the previous steps into

$$
\begin{aligned}
\mathrm{D}_{0}[f]+\mathrm{D}_{1}[f] \leq & \left(\varepsilon_{1}-D\right)\|(1-\Pi) f\|_{0}^{2}+\varepsilon_{1} \lambda_{2}\|(1-\Pi) f\|_{0}\|\Pi f\|_{0} \\
- & \varepsilon_{1} \gamma_{2}\left(\|\Pi f\|_{0}^{2}-M_{f}^{2}\right)+2 \kappa \lambda_{1}\|f\|_{0}^{2} \\
= & -\left(D-\varepsilon_{1}-2 \kappa \lambda_{1}\right)\|(1-\Pi) f\|_{0}^{2}+\varepsilon_{1} \lambda_{2}\|(1-\Pi) f\|_{0}\|\Pi f\|_{0} \\
- & \left(\varepsilon_{1} \gamma_{2}-2 \kappa \lambda_{1}\right)\|\Pi f\|_{0}^{2}+\varepsilon_{1} \gamma_{2} M_{f}^{2} \\
\leq & -\left(D-\varepsilon_{1}-2 \kappa \lambda_{1}-\frac{\varepsilon_{1} \lambda_{2} b}{2}\right)\|(1-\Pi) f\|_{0}^{2} \\
& -\left(\varepsilon_{1} \gamma_{2}-2 \kappa \lambda_{1}-\frac{\varepsilon_{1} \lambda_{2}}{2 b}\right)\|\Pi f\|_{0}^{2}+\varepsilon_{1} \gamma_{2} M_{f}^{2} \\
\leq & -2 \xi(\kappa)\|f\|_{0}^{2}+\varepsilon_{1} \gamma_{2} M_{f}^{2}
\end{aligned}
$$

by Young's inequality with the choice $b=\lambda_{2} / \gamma_{2}$, and where we used the fact that $\|(1-\Pi) f\|_{0}^{2}+\|\Pi f\|_{0}^{2}=\|f\|_{0}^{2}$. Here, $\xi(\kappa)$ is explicit, and given by

$$
\begin{aligned}
\xi(\kappa) & :=\frac{1}{2} \min \left\{D-\varepsilon_{1}\left(1+\frac{\lambda_{2}^{2}}{2 \gamma_{2}}\right), \frac{\varepsilon_{1} \gamma_{2}}{2}\right\}-\kappa \lambda_{1} \\
& =\frac{D \gamma_{2}^{2}}{2\left(\gamma_{2}^{2}+2 \gamma_{2}+\lambda_{2}^{2}\right)}-\kappa \lambda_{1}
\end{aligned}
$$

Copyright $@$ by SIAM. Unauthorized reproduction of this article is prohibited. 
since the minimum in the first term is realized when the two arguments are equal, fixing $\varepsilon_{1}=2 D \gamma_{2} /\left(\gamma_{2}^{2}+2 \gamma_{2}+\lambda_{2}^{2}\right)$. Note that this choice of $\varepsilon_{1}$ satisfies $\varepsilon_{1}<D$ and $\varepsilon_{1}<1$. Choosing $\kappa$ small enough ensures $\xi(\kappa)>0$. From this analysis we conclude

$$
\mathrm{D}_{0}[f]+\mathrm{D}_{1}[f] \leq-2 \xi(\kappa)\|f\|_{0}^{2}+\varepsilon_{1} \gamma_{2} M_{f}^{2} .
$$

Let us now add the control of $\mathrm{D}_{2}+\mathrm{D}_{3}$. If $\nabla_{x} V$ is bounded, $g \equiv 0$ and $\mathrm{D}_{3}=0$ :

$$
\begin{aligned}
\frac{\mathrm{d}}{\mathrm{d} t} \mathrm{G}[f] & =\mathrm{D}_{0}[f]+\mathrm{D}_{1}[f]+\mathrm{D}_{2}[f] \\
& \leq-\left(4 \xi(\kappa)-\kappa\left\|\nabla_{x} V\right\|_{\infty}\right) \frac{1}{2}\|f\|_{\kappa}^{2}+\varepsilon_{1} \gamma_{2} M_{f}^{2} \\
& \leq-\gamma_{1} \mathrm{G}[f]+\varepsilon_{1} \gamma_{2} M_{f}^{2}
\end{aligned}
$$

by the norm equivalence (9). Here, we defined

$$
\gamma_{1}:=\frac{4 \xi(\kappa)-\kappa\left\|\nabla_{x} V\right\|_{\infty}}{1+\varepsilon_{1}}>0 .
$$

When $\nabla_{x} V$ is unbounded, (13)-(16) imply

$$
\begin{aligned}
\frac{\mathrm{d}}{\mathrm{d} t} \mathrm{G}[f]= & \mathrm{D}_{0}[f]+\mathrm{D}_{1}[f]+\mathrm{D}_{2}[f]+\mathrm{D}_{3}[f] \\
\leq & -2 \xi(\kappa)\|f\|_{0}^{2}+\varepsilon_{1} \gamma_{2} M_{f}^{2}+\frac{\kappa}{2} C_{3} \int_{\mathbb{R}^{2} \times \mathbb{S}^{1}} f^{2} g \frac{\mathrm{d} x \mathrm{~d} \alpha}{2 \pi} \\
& +\frac{\kappa \zeta}{2}\left(C_{4}(R)\|f\|_{0}^{2}-c \int_{\mathbb{R}^{2} \times \mathbb{S}^{1}} f^{2} g \frac{\mathrm{d} x \mathrm{~d} \alpha}{2 \pi}\right) \\
= & -\frac{1}{2}\left(4 \xi(\kappa)-\kappa \zeta C_{4}(R)\right)\|f\|_{0}^{2}-\frac{\kappa \zeta}{2}\left(c-\frac{C_{3}}{\zeta}\right) \int_{\mathbb{R}^{2} \times \mathbb{S}^{1}} f^{2} g \frac{\mathrm{d} x \mathrm{~d} \alpha}{2 \pi}+\varepsilon_{1} \gamma_{2} M_{f}^{2} \\
\leq & -\frac{1}{2} \min \left\{4 \xi(\kappa)-\kappa \zeta C_{4}(R), c-\frac{C_{3}}{\zeta}\right\}\|f\|_{\kappa}^{2}+\varepsilon_{1} \gamma_{2} M_{f}^{2} \\
\leq & -\gamma_{1} \mathrm{G}[f]+\varepsilon_{1} \gamma_{2} M_{f}^{2}
\end{aligned}
$$

again by norm equivalence (9), and where we defined

$$
\gamma_{1}:=\frac{1}{1+\varepsilon_{1}} \min \left\{4 \xi(\kappa)-\kappa \zeta C_{4}(R), c-\frac{C_{3}}{\zeta}\right\}>0 .
$$

This requires $\zeta>0$ to be large enough, and the upper bound for $\kappa$ should be chosen accordingly:

$$
\zeta>\frac{C_{3}}{c}, \quad 4 \xi(\kappa)-\kappa \zeta C_{4}(R)>0 .
$$

In order to maximize the rate of convergence to equilibrium given $\kappa, D$, and $V$, one can optimize $\gamma_{1}$ over $\zeta$ while respecting the above constraints.

REMARK 2.4. The condition $\gamma_{1}>0$ translates into an explicit upper bound on $\kappa$. More precisely, we require $\xi(\kappa)>\kappa u / 4$, where $u:=\left\|\nabla_{x} V\right\|_{\infty}$ in the case of a bounded potential gradient, and $u:=\zeta C_{4}(R)$ otherwise. This condition is satisfied for small enough $\kappa$ :

$$
0 \leq \kappa<\frac{\varepsilon_{1} \gamma_{2}}{\left(4 \lambda_{1}+u\right)}=\frac{2 D \gamma_{2}^{2}}{\left(4 \lambda_{1}+u\right)\left(\gamma_{2}^{2}+2 \gamma_{2}+\lambda_{2}^{2}\right)}
$$

Copyright (c) by SIAM. Unauthorized reproduction of this article is prohibited. 
which also implies $\xi(\kappa)>0$. Recall that Proposition 1.3 requires $\kappa<1 / 3$ in the case of unbounded potential gradients. These conditions provide a range of $\kappa$ for which Proposition 2.1 holds.

3. The coercivity weight $g$. In this section, we define the function $g$ in such a way that it allows us to control the loss of weight in the perturbation operator $\mathrm{P}_{\kappa}$. When $\nabla_{x} V$ is bounded, we do not need any extra weight since then we may control the perturbation thanks to the stationary weight $e^{V}$, and so we set $g \equiv 0$ in that case. When it is not, Proposition 1.3 provides a suitable weight function $g$ by constructive methods.

3.1. Proof of Proposition 1.3. The proof is strongly inspired by [12], but our weight is different since we work in an $L^{2}$-framework rather than in an $L^{1}$ one. Assuming $\nabla_{x} V$ is unbounded, we seek a weight $g$ of the form

$$
g(x, \alpha)=\exp \left(\beta V(x)+\left|\nabla_{x} V(x)\right| \Gamma\left(\tau(\alpha) \cdot \frac{\nabla_{x} V(x)}{\left|\nabla_{x} V(x)\right|}\right)\right),
$$

where the parameter $\beta>1$ and the function $\Gamma \in C^{1}([-1,1]), \Gamma>0$ are to be determined. We define

$$
Y(x, \alpha):=\tau(\alpha) \cdot \frac{\nabla_{x} V(x)}{\left|\nabla_{x} V(x)\right|}, \quad Y^{\perp}(x, \alpha):=\tau^{\perp}(\alpha) \cdot \frac{\nabla_{x} V(x)}{\left|\nabla_{x} V(x)\right|}
$$

and split the proof into four steps: (1) we rewrite statement (6) using the explicit expression of the weight $g$, (2) we simplify the obtained expression using assumption (H5), (3) we prove the equivalent statement obtained in Step 2 by defining a suitable choice of $\Gamma(\cdot)$ and $\beta$, and (4) we demonstrate that it is indeed possible to choose suitable parameters for the calculations in Step 3 to hold, fixing explicit expressions where possible.

Step 1: Rewriting the weight estimate (6).

Applying the operator $\mathcal{L}_{\kappa}$ defined in (7) to $g$, we can compute explicitly

$$
\begin{aligned}
\frac{\mathcal{L}_{\kappa}(g)}{g}= & D\left(\left|\nabla_{x} V\right| \partial_{\alpha \alpha} \Gamma(Y)+\left|\nabla_{x} V\right|^{2}\left|\partial_{\alpha} \Gamma(Y)\right|^{2}\right) \\
& +\left(\tau(\alpha)+\kappa e_{1}\right) \cdot\left(\beta \nabla_{x} V+\nabla_{x}\left(\left|\nabla_{x} V\right| \Gamma(Y)\right)\right) \\
& -\left|\nabla_{x} V\right|^{2} Y^{\perp} \partial_{\alpha} \Gamma(Y)-\left|\nabla_{x} V\right| Y
\end{aligned}
$$

Since

$$
\partial_{\alpha} \Gamma=Y^{\perp} \Gamma^{\prime}(Y) \quad \text { and } \quad \partial_{\alpha \alpha} \Gamma=\partial_{\alpha}\left(Y^{\perp} \Gamma^{\prime}(Y)\right)=-Y \Gamma^{\prime}(Y)+\left|Y^{\perp}\right|^{2} \Gamma^{\prime \prime}(Y),
$$

we get

$$
\begin{aligned}
\frac{\mathcal{L}_{\kappa}(g)}{g}= & D\left(\left|\nabla_{x} V\right|\left(-Y \Gamma^{\prime}(Y)+\left|Y^{\perp}\right|^{2} \Gamma^{\prime \prime}(Y)\right)+\left|\nabla_{x} V\right|^{2}\left|Y^{\perp}\right|^{2}\left(\Gamma^{\prime}(Y)\right)^{2}\right) \\
& +\left(\tau(\alpha)+\kappa e_{1}\right) \cdot\left(\beta \nabla_{x} V+\nabla_{x}\left(\left|\nabla_{x} V\right| \Gamma(Y)\right)\right) \\
& -\left|\nabla_{x} V\right|^{2}\left|Y^{\perp}\right|^{2} \Gamma^{\prime}(Y)-\left|\nabla_{x} V\right| Y \\
= & \left(\beta-1-D \Gamma^{\prime}(Y)\right)\left|\nabla_{x} V\right| Y+\kappa \beta e_{1} \cdot \nabla_{x} V+\left(\tau(\alpha)+\kappa e_{1}\right) \cdot \nabla_{x}\left(\left|\nabla_{x} V\right| \Gamma(Y)\right) \\
& +\left|Y^{\perp}\right|^{2}\left(D\left|\nabla_{x} V\right| \Gamma^{\prime \prime}(Y)+\left|\nabla_{x} V\right|^{2}\left[D\left(\Gamma^{\prime}(Y)\right)^{2}-\Gamma^{\prime}(Y)\right]\right) .
\end{aligned}
$$

Copyright (c) by SIAM. Unauthorized reproduction of this article is prohibited. 
In order to see which $\Gamma$ to choose, let us divide by $\left|\nabla_{x} V\right|$ and denote the diffusion and transport part by

$$
\operatorname{diff}(x, \alpha):=\left(\tau(\alpha)+\kappa e_{1}\right) \cdot \frac{\nabla_{x}\left(\left|\nabla_{x} V\right| \Gamma(Y)\right)}{\left|\nabla_{x} V\right|}, \quad \operatorname{tran}(x):=\frac{e_{1} \cdot \nabla_{x} V}{\left|\nabla_{x} V\right|} .
$$

Now, we can rewrite the statement of Proposition 1.3: we seek a positive constant $c>0$ and a radius $R>0$ such that for any $\alpha \in \mathbb{S}^{1}$ and $|x|>R$,

$$
\begin{aligned}
\left(\beta-1-D \Gamma^{\prime}(Y)\right) Y & +\kappa \beta \operatorname{tran}(x)+\operatorname{diff}(x, \alpha) \\
+ & \left|Y^{\perp}\right|^{2}\left(D \Gamma^{\prime \prime}(Y)+\left|\nabla_{x} V\right|\left[D\left(\Gamma^{\prime}(Y)\right)^{2}-\Gamma^{\prime}(Y)\right]\right) \leq-c .
\end{aligned}
$$

To achieve this bound, note that $|Y| \leq 1$ and $|\operatorname{tran}| \leq 1$ for all $(x, \alpha) \in \mathbb{R}^{2} \times \mathbb{S}^{1}$.

Step 2: Simplifying the weight estimate.

Further, the diffusion term $\operatorname{diff}(\cdot)$ can be made arbitrarily small outside a sufficiently large ball. Indeed,

$$
\operatorname{diff}(x, \alpha)=\left(\tau+\kappa e_{1}\right) \cdot\left[\Gamma^{\prime}(Y) \nabla_{x} Y+\Gamma(Y) \frac{\nabla_{x}\left(\left|\nabla_{x} V\right|\right)}{\left|\nabla_{x} V\right|}\right],
$$

and both $\left|\nabla_{x} Y\right|$ and $\left|\nabla_{x}\left(\left|\nabla_{x} V\right|\right)\right| /\left|\nabla_{x} V\right|$ converge to zero as $|x| \rightarrow \infty$ by assumption (H5), and $\Gamma$ is bounded. In other words, using the fact that the potential gradient is unbounded, it remains to show that we can find constants $\gamma>\kappa \beta>0$ and a radius $r_{1}>0$ such that

$$
\forall|x|>r_{1}, \quad\left(\beta-1-D \Gamma^{\prime}\right) Y+\left|Y^{\perp}\right|^{2}\left(D \Gamma^{\prime \prime}+\left|\nabla_{x} V\right|\left[D\left(\Gamma^{\prime}\right)^{2}-\Gamma^{\prime}\right]\right) \leq-\gamma .
$$

Then we can choose $r_{2}>0$ such that

$$
|x|>r_{2} \Longrightarrow \forall \alpha \in \mathbb{S}^{1}, \quad \operatorname{diff}(x, \alpha) \leq \frac{\gamma-\kappa \beta}{2},
$$

and we conclude for the statement of Proposition 1.3 with $R:=\max \left\{r_{1}, r_{2}\right\}$ and $c:=(\gamma-\kappa \beta) / 2>0$.

Step 3: Proof of the weight estimate.

Proving (17) can be done by an explicit construction. We define $\Gamma^{\prime} \in C^{0}([-1,1])$ piecewise,

$$
\Gamma^{\prime}(Y)= \begin{cases}\delta^{+} & \text {if } Y>\varepsilon_{0}, \\ \frac{\delta^{+}-\delta^{-}}{2 \varepsilon_{0}}\left(Y+\varepsilon_{0}\right)+\delta^{-} & \text {if }|Y| \leq \varepsilon_{0}, \\ \delta^{-} & \text {if } Y<-\varepsilon_{0},\end{cases}
$$

where $0<\delta^{-}<\delta^{+}<1 / D$ and $\varepsilon_{0} \in(0,1)$ are to be determined (see Figure 1 ). With this choice of $\Gamma^{\prime}$, we can ensure that $\Gamma$ is strictly positive in the interval $[-1,1]$. Now, let us show that there exist suitable choices of $\gamma$ and $\beta$ for the bound (17) to hold. More precisely, we choose a suitable $\beta$ such that $(\beta-1) / D \in\left(\delta^{-}, \delta^{+}\right)$and $0<\gamma<\tilde{\gamma}$, defining $\gamma:=\varepsilon_{0}\left(1+D \delta^{+}-\beta\right)$ and $\tilde{\gamma}:=\varepsilon_{0}\left(\beta-1-D \delta^{-}\right)$. We split our analysis into cases:

- Assume $Y>\varepsilon_{0}$. Then the left-hand side (LHS) of (17) can be bounded as follows:

$$
\left(\beta-1-D \delta^{+}\right) Y+\delta^{+}\left(D \delta^{+}-1\right)\left|\nabla_{x} V \| Y^{\perp}\right|^{2}<\left(\beta-1-D \delta^{+}\right) \varepsilon_{0}=-\gamma .
$$

Copyright $@$ by SIAM. Unauthorized reproduction of this article is prohibited. 


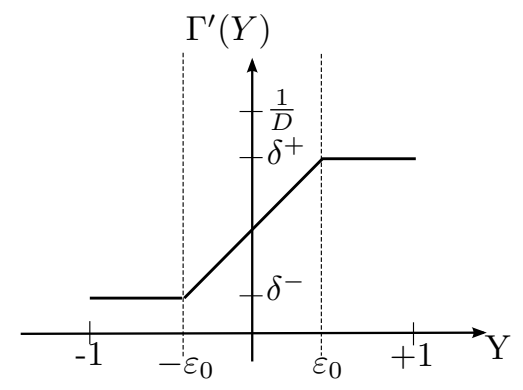

FIG. 1. Derivative of $\Gamma$.

- Assume $Y<-\varepsilon_{0}$. Then the LHS of (17) can be bounded as follows:

$$
\left(\beta-1-D \delta^{-}\right) Y+\delta^{-}\left(D \delta^{-}-1\right)\left|\nabla_{x} V \| Y^{\perp}\right|^{2}<-\left(\beta-1-D \delta^{-}\right) \varepsilon_{0}=-\tilde{\gamma} .
$$

- Assume $|Y| \leq \varepsilon_{0}$. Since $1=|Y|^{2}+\left|Y^{\perp}\right|^{2}$, we have $\left|Y^{\perp}\right|^{2} \geq 1-\varepsilon_{0}^{2}$. Further, setting

$$
h=a Y+b \in\left(\delta^{-}, \delta^{+}\right), \quad a:=\frac{\delta^{+}-\delta^{-}}{2 \varepsilon_{0}}, \quad b:=\frac{\delta^{+}+\delta^{-}}{2},
$$

we have $\Gamma^{\prime}=h$ and $D h^{2}-h \leq D \delta^{-}\left(\delta^{+}-1 / D\right)$. Now, using the fact that the potential gradient is unbounded, we can find a radius $r_{1}>0$ large enough such that for all $|x|>r_{1}$,

$$
\frac{D\left(\delta^{+}-\delta^{-}\right)}{2 \varepsilon_{0}}-D \delta^{-}\left(\frac{1}{D}-\delta^{+}\right)\left|\nabla_{x} V\right|<-\frac{2 \tilde{\gamma}}{\left(1-\varepsilon_{0}^{2}\right)} .
$$

Putting these estimates together, we obtain for $|x|>r_{1}$,

$$
\begin{aligned}
& (\beta-1-D h) Y+\left|Y^{\perp}\right|^{2}\left(\frac{D\left(\delta^{+}-\delta^{-}\right)}{2 \varepsilon_{0}}+\left|\nabla_{x} V\right|\left[D h^{2}-h\right]\right) \\
& \leq\left(\beta-1-D \delta^{-}\right) \varepsilon_{0}+\left|Y^{\perp}\right|^{2}\left(\frac{D\left(\delta^{+}-\delta^{-}\right)}{2 \varepsilon_{0}}+\left|\nabla_{x} V\right|\left[D \delta^{-}\left(\delta^{+}-\frac{1}{D}\right)\right]\right) \\
& \leq \tilde{\gamma}+\left(1-\varepsilon_{0}^{2}\right)\left(\frac{D\left(\delta^{+}-\delta^{-}\right)}{2 \varepsilon_{0}}+\left|\nabla_{x} V\right|\left[D \delta^{-}\left(\delta^{+}-\frac{1}{D}\right)\right]\right) \leq-\tilde{\gamma} .
\end{aligned}
$$

Step 4: Choice of parameters.

We now come back to the choice of $\delta^{-}, \delta^{+}, \varepsilon_{0}, \beta$ such that $\kappa \beta<\gamma$ and $0<\gamma<\tilde{\gamma}$ hold true. More precisely, these two constraints translate into the following bound on $\beta$ :

$$
1+D\left(\frac{\delta^{+}+\delta^{-}}{2}\right)<\beta<\left(\frac{\varepsilon_{0}}{\kappa+\varepsilon_{0}}\right)\left(1+D \delta^{+}\right) .
$$

It is easy to see that this bound also implies $1+D \delta^{-}<\beta<1+D \delta^{+}$as required. However, for this to be possible we need to choose $\varepsilon_{0}$ such that $L H S<R H S$, in other words,

$$
\kappa\left(\frac{2+D\left(\delta^{+}+\delta^{-}\right)}{D\left(\delta^{+}-\delta^{-}\right)}\right)<\varepsilon_{0}
$$

Copyright (C) by SIAM. Unauthorized reproduction of this article is prohibited. 
Since $\varepsilon_{0}$ has to be less than 1 and $D\left(\delta^{+}-\delta^{-}\right) /\left(2+D\left(\delta^{+}+\delta^{-}\right)\right)<1 / 3$, this bound is only possible if $\kappa \in(0,1 / 3)$; then it remains to choose $0<\delta^{-}<\delta^{+}<1 / D$ such that

$$
\kappa<\frac{D\left(\delta^{+}-\delta^{-}\right)}{2+D\left(\delta^{+}+\delta^{-}\right)} \in\left(0, \frac{1}{3}\right)
$$

To satisfy all these constraints, we make the choice of parameters (for $\kappa<1 / 3$ )

$$
\delta^{+}:=\frac{3(1+\kappa)}{4 D}, \quad \delta^{-}:=\frac{(1-3 \kappa)}{4 D} .
$$

Then (20) holds true, and we can fix $\varepsilon_{0} \in(0,1)$ to satisfy (19):

$$
\varepsilon_{0}:=\frac{1}{2}\left(1+\kappa\left(\frac{2+D\left(\delta^{+}+\delta^{-}\right)}{D\left(\delta^{+}-\delta^{-}\right)}\right)\right)=\frac{1}{2}\left(\frac{1+9 \kappa}{1+3 \kappa}\right) .
$$

Finally, we choose $\beta$ satisfying (18) as follows:

$$
\begin{aligned}
\beta & :=\frac{1}{2}\left[1+D\left(\frac{\delta^{+}+\delta^{-}}{2}\right)+\left(\frac{\varepsilon_{0}}{\kappa+\varepsilon_{0}}\right)\left(1+D \delta^{+}\right)\right] \\
& =\frac{3}{4}+\frac{(1+9 \kappa)(7+3 \kappa)}{8\left(6 \kappa^{2}+11 \kappa+1\right)} \in(1,2) .
\end{aligned}
$$

\section{Existence and uniqueness of a steady state.}

4.1. Existence of a $\mathcal{C}_{0}$-semigroup. The proof of the next theorem relies on the a priori estimates from section 2 .

Theorem 4.1. The linear operator $\mathrm{L}_{\kappa}: \mathcal{D}\left(\mathrm{L}_{\kappa}\right) \rightarrow L^{2}\left(\mathrm{~d} \mu_{\kappa}\right)$ defined in (5) is the infinitesimal generator of a $\mathcal{C}_{0}$-semigroup $\left(S_{t}\right)_{t \geq 0}$ on $L^{2}\left(\mathrm{~d} \mu_{\kappa}\right)$.

Proof. Let us denote by $\mathrm{L}_{\kappa}^{*}$ the adjoint of $\mathrm{L}_{\kappa}$ in $L^{2}\left(\mathrm{~d} \mu_{\kappa}\right)$. Both domains $\mathcal{D}\left(\mathrm{L}_{\kappa}\right)$ and $\mathcal{D}\left(\mathrm{L}_{\kappa}^{*}\right)$ contain the core $\mathcal{C}$ and are dense. The operator $\mathrm{L}_{\kappa}$ is closable in $L^{2}\left(\mathrm{~d} \mu_{\kappa}\right)$. To see this, take a sequence $\left(f_{n}\right)_{n \in \mathbb{N}} \in \mathcal{D}\left(\mathrm{L}_{\kappa}\right)$ converging to zero in $L^{2}\left(\mathrm{~d} \mu_{\kappa}\right)$ such that the sequence $\left(\mathrm{L}_{\kappa} f_{n}\right)_{n \in \mathbb{N}}$ converges to some limit $h \in L^{2}\left(\mathrm{~d} \mu_{\kappa}\right)$. Then for any test function $\varphi \in \mathcal{C}$,

$$
\left\langle\varphi, \mathrm{L}_{\kappa} f_{n}\right\rangle_{\kappa}=\left\langle\mathrm{L}_{\kappa}^{*} \varphi, f_{n}\right\rangle_{\kappa} \rightarrow 0 \quad \text { as } n \rightarrow \infty .
$$

Since the LHS converges to $\langle\varphi, h\rangle_{\kappa}$ for all $\varphi \in \mathcal{C}$, we conclude $h \equiv 0$ a.e., and so $\mathrm{L}_{\kappa}$ is closable. Similarly, $L_{\kappa}^{*}$ is closable. We denote by $\tilde{L}_{\kappa}$ and $\tilde{L}_{\kappa}^{*}$ some closed extensions of $L_{\kappa}$ and $L_{\kappa}^{*}$, respectively. The Lumer-Phillips theorem in the form [16, Corollary 4.4] states that an operator $\tilde{L}$ generates a $\mathcal{C}_{0}$-semigroup if $\tilde{L}$ is closed and both $\tilde{L}$ and $\tilde{\mathrm{L}}^{*}$ are dissipative. Since the core $\mathcal{C}$ is dense in both $\mathcal{D}\left(\tilde{\mathrm{Q}}_{\kappa}\right)$ and $\mathcal{D}\left(\tilde{\mathrm{Q}}_{\kappa}^{*}\right)$, which in turn are both dense in $L^{2}\left(\mathrm{~d} \mu_{\kappa}\right)$, then for any constant $C>0, \tilde{\mathrm{L}}_{\kappa}-C$ ld is dissipative if and only if $\tilde{L}_{\kappa}^{*}-C$ ld is dissipative. Therefore, it remains to show that $\tilde{L}_{\kappa}-C$ ld is dissipative for some $C>0$. Since the restriction of $\tilde{\mathrm{L}}_{\kappa}$ to $\mathcal{C}$ is $\mathrm{L}_{\kappa}$, it is enough to prove that $\mathrm{L}_{\kappa}-C$ ld is dissipative on $\mathcal{C}$ for some constant $C>0$. The estimates in section 2 show that there exists $C>0$ s.t.

$$
\forall f \in \mathcal{C}, \quad\left\langle\mathrm{L}_{\kappa} f, f\right\rangle_{\kappa} \leq C\|f\|_{\kappa}^{2}
$$

for some explicit constant $C>0$, which concludes the proof.

Copyright (C) by SIAM. Unauthorized reproduction of this article is prohibited. 
4.2. Proof of Theorem 1.4. Proposition 2.1 is the key ingredient to deduce the existence of a unique steady state. The set

$$
\mathcal{B}:=\left\{f \in L^{2}\left(\mathrm{~d} \mu_{\kappa}\right): \mathrm{G}[f] \leq \frac{\gamma_{2}}{\gamma_{1}}, f \geq 0, M_{f}=1\right\}
$$

is convex and bounded in $L^{2}\left(\mathrm{~d} \mu_{\kappa}\right)$ by the norm equivalence (9). By Theorem 4.1, the operator $\mathrm{L}_{\kappa}$ generates a $\mathcal{C}_{0}$-semigroup $\left(S_{t}\right)_{t \geq 0}$. Then let us show that $\mathcal{B}$ is invariant under the action of $\left(S_{t}\right)_{t \geq 0}$. Integrating in time the hypocoercivity estimate (10) in Proposition 2.1 for any $f_{\text {in }} \in L^{2}\left(\mathrm{~d} \mu_{\kappa}\right)$ with mass 1 , we obtain the bound

$$
\mathrm{G}[f(t)] \leq \mathrm{G}\left[f_{\mathrm{in}}\right] e^{-\gamma_{1} t}+\frac{\gamma_{2}}{\gamma_{1}}\left(1-e^{-\gamma_{1} t}\right)
$$

and thus

$$
\forall t>0, \quad \mathrm{G}[f(t)] \leq \max \left\{\mathrm{G}\left[f_{\text {in }}\right], \frac{\gamma_{2}}{\gamma_{1}}\right\} .
$$

Since in addition, $\left(S_{t}\right)_{t \geq 0}$ conserves mass and positivity, we conclude $S_{t}(\mathcal{B}) \subset \mathcal{B}$ for all times.

Integrate again the hypocoercivity estimate (10) in Proposition 2.1, now for the difference of two solutions with same mass, to get

$$
\mathrm{G}\left[S_{t} f-S_{t} h\right] \leq e^{-\gamma_{1} t} \mathrm{G}[f-h]
$$

for any $t>0$ and $f, h \in \mathcal{B}$. It follows by Banach's fixed-point theorem that there exists a unique $u^{t} \in \mathcal{B}$ such that $S_{t}\left(u^{t}\right)=u^{t}$ for each $t>0$. Let $t_{n}:=2^{-n}, n \in \mathbb{N}$, and $u_{n}:=u^{t_{n}}$. Then $S_{2^{-n}}\left(u_{n}\right)=u_{n}$, and by repeatedly applying the semigroup property,

$$
\forall k \in \mathbb{N}, \forall m \leq n \in \mathbb{N}, \quad S_{k 2^{-m}}\left(u_{n}\right)=u_{n} .
$$

Let us prove that $\mathcal{B}$ is weakly compact in $L^{2}\left(\mathrm{~d} \mu_{\kappa}\right)$. Consider a sequence $\left(f_{n}\right)_{n \in \mathbb{N}} \in$ $\mathcal{B}$. It has a cluster point $f$ for the weak convergence since $\mathcal{B}$ is bounded in $L^{2}\left(\mathrm{~d} \mu_{\kappa}\right)$, and the corresponding subsequence is still denoted $f_{n}$ for simplicity. By lower semicontinuity of the equivalent norm $\mathrm{G}$,

$$
\mathrm{G}[f] \leq \liminf _{n \rightarrow \infty} \mathrm{G}\left[f_{n}\right] \leq \gamma_{2} / \gamma_{1} .
$$

Further, since $f_{n} \geq 0$ for all $n \in \mathbb{N}$, it follows that $f \geq 0$. (The set of nonnegative functions is a strongly closed convex set, hence weakly closed.) It remains to show that the limit $f$ has mass 1 by preventing loss of mass at infinity. Use the Cauchy-Schwarz inequality and the norm equivalence (9) to get for $r>0$

$$
\begin{aligned}
(1+\kappa \zeta)\left(\int_{|x|>r} \Pi f_{n} d x\right)^{2} \leq & \left(\int_{|x|>r} \int_{\mathbb{S}^{1}} f_{n}^{2} e^{V} \frac{\mathrm{d} x \mathrm{~d} \alpha}{2 \pi}\right)\left(\int_{|x|>r} \int_{\mathbb{S}^{1}} e^{-V} \frac{\mathrm{d} x \mathrm{~d} \alpha}{2 \pi}\right) \\
& +\kappa \zeta\left(\int_{|x|>r} \int_{\mathbb{S}^{1}} f_{n}^{2} g \frac{\mathrm{d} x \mathrm{~d} \alpha}{2 \pi}\right)\left(\int_{|x|>r} \int_{\mathbb{S}^{1}} g^{-1} \frac{\mathrm{d} x \mathrm{~d} \alpha}{2 \pi}\right) \\
\leq & \left\|f_{n}\right\|_{\kappa}^{2}\left(\int_{|x|>r} \int_{\mathbb{S}^{1}}\left(e^{-V}+g^{-1}\right) \frac{\mathrm{d} x \mathrm{~d} \alpha}{2 \pi}\right) \\
\leq & \left(\frac{2}{1-\varepsilon_{1}}\right) \frac{\gamma_{2}}{\gamma_{1}}\left(\int_{|x|>r} \int_{\mathbb{S}^{1}} 2 e^{-V} \frac{\mathrm{d} x \mathrm{~d} \alpha}{2 \pi}\right) .
\end{aligned}
$$

Copyright $@$ by SIAM. Unauthorized reproduction of this article is prohibited. 
This shows that

$$
\begin{aligned}
& \sup _{n \in \mathbb{N}}\left(\int_{|x|>r} \Pi f_{n} d x\right) \\
& \quad \leq\left(\left(\frac{4}{\left(1-\varepsilon_{1}\right)(1+\kappa \zeta)}\right)\left(\frac{\gamma_{2}}{\gamma_{1}}\right)\right)^{1 / 2}\left(\int_{|x|>r} \int_{\mathbb{S}^{1}} e^{-V} \frac{\mathrm{d} x \mathrm{~d} \alpha}{2 \pi}\right)^{1 / 2} \rightarrow 0 \quad \text { as } r \rightarrow \infty,
\end{aligned}
$$

since $\int_{\mathbb{R}^{2} \times \mathbb{S}^{1}} e^{-V} \frac{\mathrm{d} x \mathrm{~d} \alpha}{2 \pi}=1$. Together with $M_{f_{n}}=1$ for all $n \in \mathbb{N}$, it follows that $M_{f}=1$. Hence $f \in \mathcal{B}$. The weak compactness of $\mathcal{B}$ implies the existence of a subsequence $u_{n_{j}}$ of $u_{n}$ and a function $u \in \mathcal{B}$ such that $u_{n_{j}}$ converges weakly to $u$ in $L^{2}\left(\mathrm{~d} \mu_{\kappa}\right)$. Letting $n_{j} \rightarrow \infty$ in (21) implies that (since $S_{t}$ is a continuous operator)

$$
\forall m \in \mathbb{N}, \forall k \in \mathbb{N}, \quad S_{k 2^{-m}}(u)=u .
$$

Finally the density of the dyadic rationals $\left\{k 2^{-m}: k \in \mathbb{N}, m \in \mathbb{N}\right\}$ in $(0,+\infty)$ and continuity of $S_{t}(u)$ in $t$ for all $u \in \mathcal{B}$ imply that

$$
\forall t \geq 0, \quad S_{t}(u)=u .
$$

This shows the existence and uniqueness of a global stationary state $F_{\kappa}:=u \in \mathcal{B}$.

To complete the proof of Theorem 1.4, we apply the hypocoercivity estimate Proposition 2.1 to the difference between a solution $f \in L^{2}\left(\mathrm{~d} \mu_{\kappa}\right)$ and the unique stationary state of the same mass, $M_{f} F_{\kappa}$, to show exponential convergence to equilibrium in $\|\cdot\|_{\kappa}$ : first, we deduce from the contraction estimate (10) that

$$
\mathrm{G}\left[f(t)-M_{f} F_{\kappa}\right] \leq \mathrm{G}\left[f_{\text {in }}-M_{f} F_{\kappa}\right] e^{-\gamma_{1} t},
$$

which allows us then to estimate the difference to equilibrium in the $L^{2}\left(\mathrm{~d} \mu_{\kappa}\right)$-norm. Indeed, by norm equivalence, we obtain

$$
\left\|f(t)-M_{f} F_{\kappa}\right\|_{\kappa}^{2} \leq \frac{1+\varepsilon_{1}}{1-\varepsilon_{1}}\left\|f_{\text {in }}-M_{f} F_{\kappa}\right\|_{\kappa}^{2} e^{-\gamma_{1} t} .
$$

Hence, we obtain (8) with rate of convergence $\lambda_{\kappa}:=\gamma_{1} / 2$.

5. Concluding remarks. From our previous estimates, we have that $\mathrm{G}\left(F_{k}\right)$ is uniformly bounded in $\kappa$ for $\kappa$ sufficiently small. As a consequence, $\left(F_{\kappa}\right)_{\kappa>0}$ is a relatively weakly compact family in $L^{2}\left(\mathrm{~d} \mu_{\kappa}\right)$, and by uniqueness of the stationary state in the case $\kappa=0$, we deduce that $F_{\kappa} \rightarrow F_{0}$ as $\kappa \rightarrow 0$. It could also be proved with further work that the optimal (spectral gap) relaxation rate is continuous as $\kappa \rightarrow 0$.

Working in $L^{2}\left(\mathrm{~d} \mu_{\kappa}\right) \subset L^{2}\left(\mathrm{~d} \mu_{0}\right)$ we are treating the operator $\mathrm{L}_{\kappa}$ as a small perturbation of the case $\kappa=0$ with stationary conveyor belt. The natural space to investigate the convergence to $F_{\kappa}$ in the case $\kappa>0$ however is $L^{2}\left(F_{\kappa}^{-1} \mathrm{~d} x \mathrm{~d} \alpha\right)$. In this $L^{2}$-space the transport operator $\mathrm{T}-\mathrm{P}_{\kappa}$ is not skew-symmetric and the collision operator $Q$ is not self-adjoint, so the hypocoercivity method [5] cannot be applied. To get around this, one can split the operator $L_{\kappa}$ differently into a transport and a collision part following the approach in [1]. More precisely, we can write $\mathrm{L}_{\kappa}=\tilde{Q}-\tilde{\mathrm{T}}$, where

$$
\left\{\begin{array}{l}
\tilde{\mathbf{Q}} f=\partial_{\alpha}\left(D \partial_{\alpha} f-\frac{\partial_{\alpha} F_{\kappa}}{F_{\kappa}} f\right) \\
\tilde{\mathbf{T}} f=\left(\tau+\kappa e_{1}\right) \cdot \nabla_{x} f-\partial_{\alpha}\left[\left(\tau^{\perp} \cdot \nabla_{x} V+\frac{\partial_{\alpha} F_{\kappa}}{F \kappa}\right) f\right] .
\end{array}\right.
$$

Copyright (c) by SIAM. Unauthorized reproduction of this article is prohibited. 
Then in $L^{2}\left(F_{\kappa}^{-1} \mathrm{~d} x \mathrm{~d} \alpha\right)$ the operator $\tilde{\mathrm{Q}}$ is symmetric and negative semidefinite, and the operator $\mathrm{T}$ is skew-symmetric. Furthermore, the stationary state $F_{\kappa}$ lies in the intersection of the kernels of the collision and transport operators, i.e., $F_{\kappa} \in \operatorname{Ker} \tilde{Q} \cap$ Ker $\tilde{T}$. The hypocoercivity approach requires microscopic and macroscopic coercivity of $\tilde{Q}$ and $\tilde{T}$, which then requires as in [1] to control the behavior of the stationary state at infinity, i.e., for large enough $|x|$,

$$
\forall \alpha \in \mathbb{S}^{1}, \quad e^{-\sigma_{1} V(x)} \leq F_{\kappa}(x, \alpha) \leq e^{-\sigma_{2} V(x)}
$$

for some constants $\sigma_{1}, \sigma_{2}>0$. If true, this would be an important physical information on the stationary state, but we do not know how to prove it now. Even with this information at hand, this approach requires that the existence of the stationary state is known a priori. The rate of convergence one obtains in this case may be different from the rate obtained here, and it is not clear which method yields the better rate as both are most likely not optimal.

Acknowledgments. EB is very grateful to the University of Cambridge for its sunny hospitality during the second semester of the academic year 2015-2016. The authors are very grateful to the two anonymous referees for very fruitful and detailed comments and remarks.

\section{REFERENCES}

[1] V. Calvez, G. Raoul, and C. Schmeiser, Confinement by biased velocity jumps: Aggregation of escherichia coli, Kinet. Relat. Models, 8 (2015), pp. 651-666, https://doi.org/10.3934/ krm.2015.8.651.

[2] L. Desvillettes and C. Villani, On the trend to global equilibrium in spatially inhomogeneous entropy-dissipating systems: The linear Fokker-Planck equation, Comm. Pure Appl. Math., 54 (2001), pp. 1-42, https://doi.org/10.1002/1097-0312(200101)54:1〈1::AID-CPA1〉 3.0.CO;2-Q.

[3] L. Desvillettes and C. Villani, On the trend to global equilibrium for spatially inhomogeneous kinetic systems: The Boltzmann equation, Invent. Math., 159 (2005), pp. 245-316, https://doi.org/10.1007/s00222-004-0389-9.

[4] J. Dolbeault, A. Klar, C. Mouhot, And C. Schmeiser, Exponential rate of convergence to equilibrium for a model describing fiber lay-down processes, Appl. Math. Res. Express. AMRX, 2013 (2013), pp. 165-175.

[5] J. Dolbeault, C. Mouhot, And C. Schmeiser, Hypocoercivity for linear kinetic equations conserving mass, Trans. Amer. Math. Soc., 367 (2015), pp. 3807-3828, https://doi.org/10. 1090/S0002-9947-2015-06012-7.

[6] T. Götz, A. Klar, N. Marheineke, and R. Wegener, A stochastic model and associated Fokker-Planck equation for the fiber lay-down process in nonwoven production processes, SIAM J. Appl. Math., 67 (2007), pp. 1704-1717, https://doi.org/10.1137/06067715X.

[7] M. Grothaus, A. Klar, J. Maringer, and P. Stilgenbauer, Geometry, Mixing Properties and Hypocoercivity of a Degenerate Diffusion Arising in Technical Textile Industry, arXiv/1203.4502, 2012.

[8] Y. Guo, The Landau equation in a periodic box, Comm. Math. Phys., 231 (2002), pp. 391-434, https://doi.org/10.1007/s00220-002-0729-9.

[9] F. HÉRAU AND F. NIER, Isotropic hypoellipticity and trend to equilibrium for the Fokker-Planck equation with a high-degree potential, Arch. Ration. Mech. Anal., 171 (2004), pp. 151-218, https://doi.org/10.1007/s00205-003-0276-3.

[10] A. Klar, J. Maringer, and R. Wegener, A 3D model for fiber lay-down in nonwoven production processes, Math. Models Methods Appl. Sci., 22 (2012), 1250020, https: //doi.org/10.1142/S0218202512500200.

[11] M. Kolb, M. SAvov, AND A. WüBKer, Geometric Ergodicity of a Hypoelliptic Diffusion Modelling the Melt-Spinning Process of Nonwoven Materials, arXiv/1112.6159, 2011.

[12] M. Kolb, M. Savov, AND A. WüBKER, (Non-)ergodicity of a degenerate diffusion modeling the fiber lay down process, SIAM J. Math. Anal., 45 (2013), pp. 1-13, https://doi.org/10. $1137 / 120870724$.

Copyright $@$ by SIAM. Unauthorized reproduction of this article is prohibited. 
[13] T.-P. Liu, T. YANG, AND S.-H. YU, Energy method for Boltzmann equation, Phys. D, 188 (2004), pp. 178-192, https://doi.org/10.1016/j.physd.2003.07.011.

[14] N. Marheineke and R. Wegener, Fiber dynamics in turbulent flows: General modeling framework, SIAM J. Appl. Math., 66 (2006), pp. 1703-1726, https://doi.org/10.1137/ 050637182 .

[15] N. Marheineke AND R. Wegener, Fiber dynamics in turbulent flows: Specific Taylor drag, SIAM J. Appl. Math., 68 (2007), pp. 1-23, https://doi.org/10.1137/06065489X.

[16] A. PAZY, Semigroups of Linear Operators and Applications to Partial Differential Equations, Appl. Math. Sci. 44, Springer-Verlag, New York, 1983, https://doi.org/10.1007/ 978-1-4612-5561-1.

[17] C. Villani, Hypocoercivity, Mem. Amer. Math. Soc., 202 (2009), https://doi.org/10.1090/ S0065-9266-09-00567-5.

Copyright $@$ by SIAM. Unauthorized reproduction of this article is prohibited. 